\title{
A series whose sum range is an arbitrary finite set
}

\author{
by \\ Jakub OnUfry Wojtaszczyk (Warszawa)
}

\begin{abstract}
In finite-dimensional spaces the sum range of a series has to be an affine subspace. It has long been known that this is not the case in infinite-dimensional Banach spaces. In particular in 1984 M. I. Kadets and K. Woźniakowski obtained an example of a series whose sum range consisted of two points, and asked whether it was possible to obtain more than two, but finitely many points. This paper answers this question affirmatively, by showing how to obtain an arbitrary finite set as the sum range of a series in any infinite-dimensional Banach space.
\end{abstract}

1. Introduction. For a finite-dimensional linear space $X$ the wellknown Steinitz theorem states that for any conditionally convergent series the set of all possible limits of the series (called the sum range) is an affine subspace of $X$. In the "Scottish Book" S. Banach posed the problem whether the same holds for infinite-dimensional Banach spaces. The problem was solved negatively in the same book by J. Marcinkiewicz. In his example the sum range is the set $M$ of all integer-valued functions in $L_{2}[0,1]$. The next example, due to M. I. Ostrovskii, showed that the sum range does not have to be a closed set - the sum range of Ostrovskii's series was of the form $M+\sqrt{2} M$. Finally, M. I. Kadets constructed an example in which the sum range consisted of two points, disproving, in particular, H. Hadwiger's conjecture that the sum range has to be the coset of some additive subgroup of $X$. The justification of the example was obtained independently by K. Woźniakowski and P. A. Kornilov in 1986.

It is still unknown what sets can be sum ranges of series. In this paper it is shown that any finite subset of $X$ can be the sum range of a conditionally convergent series, which solves the problem posed by M. I. Kadets along with his two-point example (the problem is stated in [S91] in the general case, and in [U02] for $X=C(\Delta)$ and $n=3)$. The example is an extension of the 2-point example of M. I. Kadets as given in [S91]. I have tried to keep the notation consistent with Kadets' notation as far as possible.

2000 Mathematics Subject Classification: 46B15, 40A30. 
All function spaces are considered with the $L_{1}$ norm, i.e. $\|f\|_{X}=$ $\int_{X}|f(x)| d x$. Frequently it is obvious on which set the integral is taken, and we just write $\|f\|$.

2. The results of K. Woźniakowski. Our work is strongly inspired by the 2-point example of M. I. Kadets and the proof by K. Woźniakowski. We will use not only the final result of Woźniakowski's work, but also multiple technical facts than can be found in the proof. Rather than force the reader to search for those in the original paper, we repeat here part of Woźniakowski's work, at times formulating the results in a way that will make them easier to use in the subsequent sections. This section is based on [S91], and a reader familiar with that work may probably skip it.

Let $Q=[0,1]^{\omega}$ be the infinite-dimensional cube, i.e. the product of a countable number of unit segments, equipped with the standard product topology and measure. By $x=\left(x_{1}, x_{2}, \ldots\right)$ we shall denote the variable ranging over $Q$. Suppose we have two sequences of functions on the cube: $a_{m}^{n}$ and $b_{m, j}^{n}$, where $n \in \mathbb{N}$, and for given $n$ the indices $m$ and $j$ belong to some finite sets $M_{n}$ and $J_{n}=M_{n+1}$ respectively. Set

$$
A_{n}=\left\{a_{m}^{n}: m \in M_{n}\right\}, \quad B_{n}=\left\{b_{m, j}^{n}: m \in M_{n}, j \in J_{n}\right\} .
$$

For convenience if $X$ is a set of functions, we shall denote by $\tilde{X}$ the sum of the functions from $X$.

We shall assume the following properties of the functions $a_{m}^{n}$ and $b_{m, j}^{n}$ :

$$
\begin{aligned}
& \tilde{A_{n}}(x)=1 \quad \forall \forall_{n \in \mathbb{N}} \forall_{x \in Q}, \\
& \left\|a_{m}^{n}\right\|=1 /\left|M_{n}\right|, \\
& \lim _{n \rightarrow \infty}\left|M_{n}\right|=\infty, \\
& a_{m}^{n} \text { depends only on the variable } x_{n}, \\
& a_{m}^{n} \text { assumes only the values } 0 \text { and } 1, \\
& b_{m, j}^{n}=-a_{m}^{n} \cdot a_{j}^{n+1} \text {. }
\end{aligned}
$$

We shall refer to these properties as the Kadets properties on the cube $Q$. They mean that for each $n$ the interval $[0,1]$ is divided into $\left|M_{n}\right|$ sets $V_{m}^{n}$ of equal measure, and $a_{m}^{n}\left(x_{1}, x_{2}, \ldots\right)=1$ iff $x_{n} \in V_{m}^{n}$. The functions $b_{m, j}^{n}$ are negative, and are supported on the rectangles $\left\{\left(x_{n}, x_{n+1}\right) \in V_{m}^{n} \times V_{j}^{n+1}\right\}$.

From the Kadets properties we can easily deduce another few properties, mainly concerning the behaviour of $b_{m, j}^{n}$, based on properties (1) and (6):

$$
\begin{aligned}
& a_{m}^{n}=-\sum_{j \in J_{n}} b_{m, j}^{n}, \\
& a_{j}^{n+1}=-\sum_{m \in M_{n}} b_{m, j}^{n},
\end{aligned}
$$


(11) $b_{m, j}^{n}$ depends only on $x_{n}$ and $x_{n+1}$,

(12) $b_{m, j}^{n}$ assumes only the values 0 and -1 ,

(13) $\quad a_{m}^{n}$ and $a_{m^{\prime}}^{n}$ have almost disjoint supports for $m \neq m^{\prime}$.

Property (13) means that the intersection of two supports is of measure zero. We can obviously modify $a_{m}^{n}$ so that the Kadets properties still hold and the sets $\left\{x: a_{m}^{n}(x)>0\right\}$ are disjoint for any constant $n$ and any two different values of $m$.

Let $c_{k}, k \in \mathbb{N}$, be any ordering of all the functions $a_{m}^{n}$ and $b_{m, j}^{n}$. Following Woźniakowski we shall investigate the convergence of any reordering $c_{\sigma(k)}$ of $c_{k}$.

Proposition 2.1. For any family of functions $c_{k}$ having the Kadets properties there exist two permutations $\sigma$ and $\tau$ of $\mathbb{N}$ such that $\sum c_{\sigma(k)}=\mathbf{0}$ and $\sum c_{\tau(k)}=\mathbf{1}$.

Proof. For $\sigma$ it is enough to order the functions $a_{m}^{n}$ lexicographically, i.e. $a_{m}^{n}$ appears before $a_{m^{\prime}}^{n^{\prime}}$ iff $n<n^{\prime}$ or $n=n^{\prime}$ and $m<m^{\prime}$, and then immediately after each $a_{m}^{n}$ we put the whole set $\left\{b_{m, j}^{n}: j \in J_{n}\right\}$. Then the sum of each block consisting of a single function $a_{m}^{n}$ and the functions $b_{m, j}^{n}$ following it is zero due to property (7), so the norm of each partial sum is the norm of the currently open block, which converges to zero by (2), (10) and (3).

To get $\tau$ we order the functions $a_{m}^{n}$ in the same way, but each function $a_{m}^{n}$ for $n>1$ is followed by the set $\left\{b_{l, m}^{n-1}: l \in M_{n-1}\right\}$, while the functions $a_{m}^{1}$ are not followed by anything (as there are no functions $b_{m, j}^{0}$ ). Then the functions $a_{m}^{1}$ sum up to the constant function 1 due to property (1). The following blocks again sum up to zero, this time by (8), so the norm of the difference between 1 and a particular partial sum is equal to the norm of the currently open block, which again converges to zero by (2), (10) and (3).

REMARK 1. The series of functions from Proposition 2.1 converge not only in the $L_{1}$ norm, but also in any $L_{p}$ norm for any $p<\infty$.

Proof. Again we only have to investigate the norm of any given block, as the sum of the previous blocks is zero. The functions $a_{m}^{n}$ assume only the values 0 and 1 and have disjoint supports for fixed $n$ from properties (5) and (13). The functions $b_{m, j}^{n}$ for given $n$ have disjoint supports (this follows from (6) and (13)) and assume the values 0 and -1 (by (12)). Thus for any $f$ which is the sum of any set of functions $a_{m}^{n}$ and $b_{m, j}^{n}$ for fixed $n$ (or $a_{m}^{n}$ and $b_{m, j}^{n-1}$ for fixed $n$ in the case of $\tau$ ) we have $\|f\|_{\infty} \leq 1$. This implies that 
for any $1 \leq p<\infty$,

$$
\begin{aligned}
\|f\|_{p} & =\left(\int|f|^{p}\right)^{1 / p}=\left(\int|f| \cdot|f|^{p-1}\right)^{1 / p} \leq\left(\|f\|_{1} \cdot\left\|f^{p-1}\right\|_{\infty}\right)^{1 / p} \\
& \leq\|f\|_{1}^{1 / p} \cdot 1=\|f\|_{1}^{1 / p} .
\end{aligned}
$$

Thus if the sum of the series tends to zero in the $L_{1}$ norm as $n \rightarrow \infty$, it also tends to zero in any $L_{p}$ norm for $p<\infty$.

Proposition 2.2. If a reordering $c_{\sigma(k)}$ of a family $c_{k}$ having the Kadets properties converges, it converges to a constant integer function.

Proof. By (4) and (11) and the finiteness of $M_{n}$ and $J_{n}$, only finitely many of the functions $c_{\sigma(k)}$ depend on a given variable $x_{l}$, precisely the functions belonging to $A_{l}, B_{l}$ and $B_{l-1}$. Moreover, their sum is the constant function -1 by (1) and (9). Thus for some integer $K_{0}$ the function $\sum_{k=1}^{K} c_{\sigma(k)}$ is constant with respect to $x_{l}$ for $K \geq K_{0}$, and thus the limit of the series also has to be constant with respect to $x_{l}$. As this holds for any $l$, the limit just has to be constant.

As the functions $c_{k}$ are integer-valued (properties (5) and (12)), so are their sums. Thus all partial sums of the series are integer-valued, and so the limit is also integer-valued, which ends the proof.

The next step will be to show that 0 and 1 are the only possible limits of a rearrangement of a family of functions with the Kadets property. We shall fix a rearrangement $c_{\sigma(k)}$ of a given Kadets family, and we shall assume that the sum $\sum_{k} c_{\sigma(k)}$ converges to some constant integer $C \neq 1$ (we know $C=1$ can be achieved); it remains to prove that under these assumptions $C=0$.

Take an arbitrary $\delta>0$ and fix $K_{0}=K_{0}(\delta)$ such that for any $K>K_{0}$,

$$
\left\|C-\sum_{k=1}^{K} c_{\sigma(k)}\right\| \leq \delta
$$

and for any $m>l>K_{0}$ the Cauchy condition holds, i.e.

$$
\left\|\sum_{k=l}^{m} c_{\sigma(k)}\right\| \leq \delta \text {. }
$$

In addition to the sets $A_{n}$ and $B_{n}$ introduced earlier we shall also consider $V_{n}=\bigcup_{k=1}^{n}\left(A_{k} \cup B_{k}\right)$. Let $M$ be any integer such that

$$
c_{\sigma(k)} \in V_{M} \cup A_{M+1} \quad \text { for any } k \leq K .
$$

Let

$$
c_{k}^{*}=\left\{\begin{array}{ll}
c_{\sigma(k)} & \text { if } c_{\sigma(k)} \in V_{M} \cup A_{M+1}, \\
0 & \text { otherwise }
\end{array} \quad \bar{c}_{k}= \begin{cases}c_{\sigma(k)} & \text { if } c_{\sigma(k)} \in B_{M+1}, \\
0 & \text { otherwise }\end{cases}\right.
$$


Define

$$
c^{*}=\sum_{k=K_{0}+1}^{\infty} c_{k}^{*}, \quad c=\sum_{k=1}^{K_{0}} c_{\sigma(k)} .
$$

Then $c+c^{*}=\tilde{V}_{M}+\tilde{A}_{M+1}=0+1=1$. Hence $\left\|c^{*}\right\|=\|1-c\| \geq\|1-C\|-$ $\|C-c\| \geq 1-\delta$. Let $k_{0}=K_{0}$ and

$$
k_{j+1}=\min \left\{k: \frac{1}{4}-\frac{5 \delta}{4} \leq\left\|\sum_{i=k_{j}+1}^{k} c_{k}^{*}\right\| \leq \frac{1}{4}-\frac{\delta}{4}\right\} .
$$

The indices $k_{j}$ are well defined for $j$ from 1 to 4 because the norm of $c^{*}$ is at least $1-\delta$ and each single $c_{k}^{*}$ has norm $\leq \delta$ by (16). For $j=0,1,2,3$ define

$$
c_{j+1}^{* *}=\sum_{k=k_{j}+1}^{k_{j+1}} c_{k}^{*}, \quad \overline{\bar{c}}_{j+1}=\sum_{k=k_{j}+1}^{k_{j+1}} \bar{c}_{k}, \quad \hat{c}_{j+1}=\sum_{k=k_{j}+1}^{k_{j+1}} c_{\sigma(k)},
$$

and for $j=1,2,3,4$ set

$$
r_{j}=\hat{c}_{j}-\bar{c}_{j}-c_{j}^{* *}
$$

In plain words this means that we divide the functions $c_{k}$ for $k_{j}<k \leq$ $k_{j+1}$ into three sets: those from $A_{n}$ for $n \leq M+1$ or $B_{n}$ for $n \leq M$ (these add up to $c_{j}^{* *}$ ), those from $B_{M+1}$ (these add up to $\overline{\bar{c}}_{j}$ ), and the rest (these add up to $r_{j}$ ). We will show that the functions from $B_{M+1}$ are placed in $c_{k}$ in similar proportions as the functions from $V_{M} \cup A_{M+1}$-if, say, about half of the functions from $V_{M} \cup A_{M+1}$ appears in $c_{k}$ (that happens at $k_{2}$ ) then about half of the functions from $B_{M+1}$ must also appear.

We shall need to estimate the norms of two sums, which we would like to be negligible: $\left\|r_{j}\right\|$ and $\left\|\sum_{k=k_{4}+1}^{\infty} c_{k}^{*}\right\|$. We know that the sum of all $c_{k}$ up to $k_{j}$ is negligible, thus if the high- $n$ functions $\left(r_{j}\right)$ are negligible, the functions from $V_{M} \cup A_{M+1}$ and $B_{M+1}$ have to approximately cancel each other out. This motivates the following proposition:

Proposition 2.3. For a Kadets family of functions $c_{k}$ with rearrangement $c_{\sigma(k)}$ converging to some $C \neq 1$, and for any $\delta$ and $M>K_{0}(\delta)$ as above,

$$
\sum_{j=1}^{4}\left\|r_{j}\right\| \leq 18 \delta
$$

Proof. As $c_{j}^{* *}$ is integer-valued (being a sum of functions from a Kadets family), the condition $\left\|c_{j}^{* *}\right\| \leq 1 / 4$ implies $\left|\operatorname{supp} c_{j}^{* *}\right| \leq 1 / 4$. Thus we can use Lemma 1 from Section 4 to get

$$
\left\|c_{j}^{* *}+r_{j}\right\| \geq\left\|c_{j}^{* *}\right\|+\left(1-2\left|\operatorname{supp} c_{j}^{* *}\right|\right)\left\|r_{j}\right\|=\left\|c_{j}^{* *}\right\|+\frac{1}{2} r_{j}
$$


Clearly $\left\|\hat{c}_{j}\right\| \leq \delta$ from the Cauchy condition (16). We thus have

$$
\begin{aligned}
1 & \geq \sum_{j=1}^{4}\left\|\overline{\bar{c}}_{j}\right\|=\sum_{j=1}^{4}\left\|\hat{c}_{j}-c_{j}^{* *}-r_{j}\right\| \geq \sum_{j=1}^{4}\left\|c_{j}^{* *}+r_{j}\right\|-\sum_{j=1}^{4}\left\|\hat{c}_{j}\right\| \\
& \geq \sum_{j=1}^{4}\left(\left\|c_{j}^{* *}\right\|+\frac{1}{2}\left\|r_{j}\right\|\right)-4 \delta \geq 1-5 \delta+\frac{1}{2} \sum_{j=1}^{4}\left\|r_{j}\right\|-4 \delta,
\end{aligned}
$$

which gives the asserted estimate. In particular, each $\left\|r_{j}\right\|$ is bounded by $18 \delta$.

COROLlary 2.4. With the notation and assumptions as above,

$$
\left\|\overline{\bar{c}}_{j}+c_{j}^{* *}\right\| \leq 19 \delta \text {. }
$$

Proof. $\left\|\overline{\bar{c}}_{j}+c_{j}^{* *}\right\|=\left\|\hat{c}_{j}-r_{j}\right\| \leq\left\|\hat{c}_{j}\right\|+\left\|r_{j}\right\| \leq \delta+18 \delta=19 \delta$.

Proposition 2.5. With the notation and assumptions as above,

$$
\left\|\sum_{k=k_{4}+1}^{\infty} c_{k}^{*}\right\| \leq 11 \delta
$$

Proof. We have

$$
\begin{aligned}
\left\|\overline{\bar{c}}_{j}\right\| & =\left\|\hat{c}_{j}-c_{j}^{* *}-r_{j}\right\| \geq\left\|c_{j}^{* *}+r_{j}\right\|-\left\|\hat{c}_{j}\right\| \geq\left\|c_{j}^{* *}\right\|+\frac{1}{2}\left\|r_{j}\right\|-\left\|\hat{c}_{j}\right\| \\
& \geq\left\|c_{j}^{* *}\right\|-\delta \geq 1 / 4-9 \delta / 4 .
\end{aligned}
$$

Suppose that $\left\|\sum_{k=k_{4}+1}^{k^{\prime}} c_{k}^{*}\right\|>11 \delta$ for some $k^{\prime}>k_{4}$. Then there would exist $k_{5} \in\left(k_{4}, k^{\prime}\right]$ such that $12 \delta \geq\left\|\sum_{k=k_{4}+1}^{k_{5}} c_{k}^{*}\right\|>11 \delta$. Then by a similar argument $\left(\left\|\overline{\bar{c}}_{5}\right\| \geq\left\|c_{5}^{* *}\right\|+(1-24 \delta)\left\|r_{5}\right\|-\left\|\hat{c}_{5}\right\| \geq 11 \delta-\delta\right)$ the norm of $\sum_{k=k_{4}+1}^{k_{5}} \bar{c}_{k}$ would be larger than $10 \delta$. But all the functions $\bar{c}_{k}$ are negative, so $\left\|\sum \bar{c}_{k}\right\|=\sum\left\|\bar{c}_{k}\right\|$, which in this case gives

$$
1 \geq\left\|\sum_{k=k_{0}}^{k_{5}} \bar{c}_{k}\right\|=\sum_{j=1}^{4}\left\|\bar{c}_{k}\right\|+\left\|\sum_{k=k_{4}+1}^{k_{5}} \bar{c}_{k}\right\|>1-9 \delta+10 \delta,
$$

a contradiction. Thus $\left\|\sum_{k=k_{4}+1}^{\infty} c_{k}^{*}\right\| \leq 11 \delta$ (the sum is convergent, as it is in fact the sum of a finite number of functions, all coming from $V_{M+1}$ ). We will denote this sum by $c_{5}^{* *}$.

Now we can prove the main theorem of Woźniakowski's work:

THEOREM 2.6. For a Kadets family of functions $c_{k}$ with some rearrangement $c_{\sigma(k)}$ converging to $C \neq 1$, we have $|C-1 / 2| \leq 1 / 2$, which (by Proposition 2.2) implies $C=0$.

Proof. Consider any $\delta$, and the partial sum $S=\sum_{k=1}^{k_{4}} c_{\sigma(k)}$ with the notation as above. As $k_{4}>K_{0}$, from assumption (15) we know that $\|S-C\|$ $\leq \delta$, so it will suffice to estimate $\|S-1 / 2\|$. We have 


$$
\begin{aligned}
& \left\|S-\frac{1}{2}\right\|=\left\|c+\sum_{j=1}^{4} c_{j}^{* *}+\sum_{j=1}^{4} \overline{\bar{c}}_{j}+\sum_{j=1}^{4} r_{j}+c_{5}^{* *}-c_{5}^{* *}-\frac{1}{2}\right\| \\
& =\left\|c+c^{*}-\frac{1}{2}+\sum_{j=1}^{4} \overline{\bar{c}}_{j}+\sum_{j=1}^{4} r_{j}-c_{5}^{* *}\right\| \leq\left\|\frac{1}{2}+\sum_{j=1}^{4} \overline{\bar{c}}_{j}\right\|+\left\|\sum_{j=1}^{4} r_{j}\right\|+\left\|c_{5}^{* *}\right\| .
\end{aligned}
$$

The function $\sum_{j=1}^{4} \overline{\bar{c}}_{j}$ is a sum of functions from $B_{M+1}$, which means it assumes only the values 0 and -1 , thus $\left|1 / 2+\sum_{j=1}^{4} \overline{\bar{c}}_{j}\right|$ is always equal to $1 / 2$. Inserting this and the bounds on $r_{j}$ and $c_{5}^{* *}$ we get

$$
\|S-1 / 2\| \leq 1 / 2+18 \delta+11 \delta=1 / 2+29 \delta .
$$

As $\|S-C\| \leq \delta$ we get $\|C-1 / 2\| \leq 1 / 2+30 \delta$. As $\delta$ is arbitrary, we get the assertion.

COROLlary 2.7. The sum range of any Kadets family consists of two points, the constant functions 0 and 1 , in any $L_{p}$ norm for $1 \leq p<\infty$.

Proof. From Proposition 2.1 and Remark 1 we know that the two constant functions belong to the sum range. From Proposition 2.2 we know that all functions in the sum range in the $L_{1}$ norm are constant integer functions, and from Theorem 2.6, only the 0 and 1 functions are eligible. If a permutation of the series converged to some function $g$ in some $L_{p}$ norm, then $\left\|S_{n}-g\right\|_{p}$ would tend to zero, where $S_{n}=\sum_{k=1}^{n} c_{\sigma(k)}$. But from the Hölder inequality we know that $\left\|S_{n}-g\right\|_{p} \geq\left\|S_{n}-g\right\|_{1}$ (as the measure of the whole space is 1 ), which would imply that the series $S_{n}$ also converges in the $L_{1}$ norm, contradicting Theorem 2.6.

3. The 3-point series. Let $Q_{i}, i=1,2,3$, be three copies of the cube $Q$. The example will be constructed in $L_{1}\left(Q_{1} \cup Q_{2} \cup Q_{3}\right)$. In the whole paper $t=\left(t_{1}, t_{2}, \ldots\right)$ will vary in $Q_{1}, u=\left(u_{1}, u_{2}, \ldots\right)$ in $Q_{2}$ and $v=\left(v_{1}, v_{2}, \ldots\right)$ in $Q_{3}$.

Our series will consist of functions of three kinds. The functions of the first kind are defined as follows:

$$
\begin{aligned}
& f_{m}^{n}(t)= \begin{cases}1 & \text { if } \frac{m-1}{n}<t_{n}<\frac{m}{n} \\
0 & \text { otherwise, }\end{cases} \\
& f_{m}^{n}(u)=f_{m}^{n}(v)=0 \quad \text { for } n \in \mathbb{N}, m \in\{1, \ldots, n\} .
\end{aligned}
$$

The second kind of functions are non-zero on all three cubes:

$$
\begin{aligned}
& g_{m, j}^{n}(t)= \begin{cases}-1 & \text { if } \frac{m-1}{n}<t_{n}<\frac{m}{n} \text { and } \frac{j-1}{n+1}<t_{n+1}<\frac{j}{n+1}, \\
0 & \text { otherwise }\end{cases} \\
& g_{m, j}^{n}(u)= \begin{cases}\frac{1}{n+1} & \text { if } \frac{m-1}{n}<u_{n}<\frac{m}{n} \\
0 & \text { otherwise }\end{cases}
\end{aligned}
$$




$$
g_{m, j}^{n}(v)= \begin{cases}1 & \text { if } \frac{(m-1)(n+1)+j-1}{n(n+1)}<v_{n}<\frac{(m-1)(n+1)+j}{n(n+1)} \\ 0 & \text { otherwise }\end{cases}
$$

for $n \in \mathbb{N}, m \in\{1, \ldots, n\}, j \in\{1, \ldots, n+1\}$.

The functions of the third kind are non-zero on $Q_{2}$ and $Q_{3}$ :

$$
\begin{aligned}
& h_{m, j, k}^{n}(t)=0, \\
& h_{m, j, k}^{n}(u)= \begin{cases}-\frac{1}{(n+1)^{2}(n+2)} & \text { if } \frac{m-1}{n}<u_{n}<\frac{m}{n}, \\
0 & \text { otherwise, }\end{cases} \\
& h_{m, j, k}^{n}(v)= \begin{cases}-1 & \text { if } \frac{(m-1)(n+1)+j-1}{n(n+1)}<v_{n}<\frac{(m-1)(n+1)+j}{n(n+1)} \\
0 & \text { and } \frac{k-1}{(n+1)(n+2)}<v_{n+1}<\frac{k}{(n+1)(n+2)},\end{cases}
\end{aligned}
$$

for $n \in \mathbb{N}, m \in\{1, \ldots, n\}, j \in\{1, \ldots, n+1\}, k \in\{1, \ldots,(n+1)(n+2)\}$.

These functions have properties we want to generalize. Suppose we have three families of indices: $M_{n}, J_{n}$ and $K_{n}$, with $J_{n}=M_{n+1}$ and $K_{n}=$ $M_{n+1} \times J_{n+1}$ (here $M_{n}=\{1, \ldots, n\}$ and the mapping from $\{1, \ldots, n+1\} \times$ $\{1, \ldots, n+2\}$ to $\{1, \ldots,(n+1)(n+2)\}$, needed to make $k$ a single index, is given by $(m, j) \mapsto(m-1)(n+1)+j)$. We have three families of functions: the first kind $\left\{f_{m}^{n}: n \in \mathbb{N}, m \in M_{n}\right\}$, the second kind $\left\{g_{m, j}^{n}: n \in \mathbb{N}, m \in M_{n}, j \in J_{n}\right\}$ and the third kind $\left\{h_{m, j, k}^{n}: n \in \mathbb{N}, m \in M_{n}, j \in J_{n}, k \in K_{n}\right\}$ defined on the union $Q_{1} \cup Q_{2} \cup Q_{3}$ of Hilbert cubes. The families $f$ and $g$ form a Kadets family on $Q_{1}$, while the functions $h$ vanish identically on $Q_{1}$. On $Q_{3}$ the functions $g$ and $h$ form a Kadets family (with $M_{n} \times J_{n}$ being the first index set and $K_{n}$ the second), while the functions $f$ vanish. The properties of the functions on $Q_{2}$ are different:

$$
\begin{aligned}
& \sum_{m \in M_{n}} \sum_{j \in J_{n}} g_{m, j}^{n}=\mathbf{1}, \\
& \sum_{m \in M_{n}} \sum_{j \in J_{n}} \sum_{k \in K_{n}} h_{m, j, k}^{n}=\mathbf{- 1}, \\
& g_{m, j}^{n}=-\sum_{k \in K_{n}} h_{m, j, k}^{n}, \\
& \sum_{m^{\prime} \in M_{n+1}} g_{m^{\prime}, j^{\prime}}^{n+1}=-\sum_{m \in M_{n}} \sum_{j \in J_{n}} \sum_{m^{\prime} \in M_{n+1}} h_{m, j,\left(m^{\prime}, j^{\prime}\right)}^{n}, \\
& \sum_{j \in J_{n}} g_{m, j} \text { assumes only the values } 0 \text { and } 1,
\end{aligned}
$$$$
\int_{Q_{2}} g_{m, j}^{n}=\int_{Q_{3}} g_{m, j}^{n}
$$ 


$$
\begin{aligned}
& \int_{Q_{2}} h_{m, j, k}^{n}=\int_{Q_{3}} h_{m, j, k}^{n}, \\
& \left\|g_{m, j}^{n}\right\|=\frac{1}{\left|M_{n} \times J_{n}\right|}, \\
& \left\|h_{m, j, k}^{n}\right\|=\frac{1}{\left|M_{n} \times J_{n} \times K_{n}\right|}, \\
& g_{m, j}^{n} \text { and } h_{m, j, k}^{n} \text { on } Q_{2} \text { depend only on } u_{n} .
\end{aligned}
$$

Such a family of functions will be called a 3-Kadets family. It is easy (although maybe a bit tedious) to check that the family defined at the beginning of this section is a 3-Kadets family.

We set

$$
\begin{gathered}
F_{n}=\left\{f_{m}^{n}: m \in M_{n}\right\}, \quad G_{n}=\left\{g_{m, j}^{n}: m \in M_{n}, j \in J_{n}\right\}, \\
H_{n}=\left\{h_{m, j, k}^{n}: m \in M_{n}, j \in J_{n}, k \in K_{n}\right\}, \quad V_{M}=\bigcup_{k=1}^{M} F_{k} \cup G_{k} \cup H_{k} .
\end{gathered}
$$

Denote by $d_{n}$ any fixed enumeration of the whole 3-Kadets family. We are investigating the possible values of $\sum_{n=1}^{\infty} d_{\sigma(n)}$ for all permutations $\sigma$ of $\mathbb{N}$.

If for a given rearrangement $\sum d_{\sigma(n)}$ converges, it converges on each of the cubes separately. On $Q_{1}$ and $Q_{3}$ we have Kadets families of functions, so on each of these cubes the series converges either to $\mathbf{0}$ or to $\mathbf{1}$ due to Theorem 2.6. The new part is the behaviour on $Q_{2}$. As in the first paragraph of the proof of Proposition 2.2, only finitely many functions depend on a given variable $u_{n}$ - the functions $g_{m, j}^{n}$ and $h_{m, j, k}^{n}$ - and their sum is constant, equal to zero by (21) applied to each $j$ separately. Thus again the sum $\sum d_{\sigma(n)}$ on $Q_{2}$ has to be a constant function.

As $\int_{Q_{2}} d_{n}=\int_{Q_{3}} d_{n}$ for any $d_{n}$ (it is 0 for functions of the first kind and follows from properties (24) and (25) for the second and third kinds), we get

$$
\int_{Q_{2}} \sum_{n=1}^{N} d_{\sigma(n)}=\int_{Q_{3}} \sum_{n=1}^{N} d_{\sigma(n)} .
$$

As the integral is a continuous functional on $L_{1}\left(Q_{2}\right)$ and $L_{1}\left(Q_{3}\right)$, the integrals of the limits have to be equal. But we know that $\sum d_{\sigma(n)}$ on both $Q_{2}$ and $Q_{3}$ is a constant function, so equality of the integrals implies equality of the sums. Thus the sum of the whole series is described by a pair of integers: the value on $Q_{1}$ and the value on $Q_{3}$. Let us denote the limit function by $d_{\infty}$.

We will show that it is possible to obtain exactly three different sums: $(\mathbf{0}, \mathbf{0}),(\mathbf{1}, \mathbf{0})$ and $(\mathbf{1}, \mathbf{1})$. To obtain any of these limits we first arrange the functions $f$ and $g$ as in Proposition 2.1 for a Kadets family on $Q_{1}$, and then after each $g$ we put the $h$ functions as in Proposition 2.1 for the cube $Q_{3}$. It remains to show convergence on $Q_{2}$. 
In the case of $(\mathbf{0}, \mathbf{0})$ after a given $f_{m}^{n}$ there appear all $g_{m, j}^{n}$ and $h_{m, j, k}^{n}$ with the same $m$ and $n$. Their sum on $Q_{2}$ is equal to $\mathbf{0}$ by (21) applied for each $j$ separately. Thus the norm of a partial sum on $Q_{2}$ is equal to the norm of the functions appearing after the last $f$, and this tends to zero by (26), (27) and (3) (all the functions have the same index $m$, so the sum of their norms is equal to $\left.2 /\left|M_{n}\right| \rightarrow 0\right)$.

In the case of $(\mathbf{1}, \mathbf{0})$ after a given $f_{m}^{n}$ we get the functions $g_{l, m}^{n-1}$ and $h_{l, m, k}^{n-1}$. Their sum on $Q_{2}$ is again $\mathbf{0}$ by (21), this time applied to each $l$ separately. Again the norm of the difference between a partial sum and $(\mathbf{1}, \mathbf{0})$ is the norm of the part after the last $f$, and that again tends to 0 .

In the case of $(\mathbf{1}, \mathbf{1})$ after a given $f_{m}^{n}$ we get the functions $g_{l, m}^{n-1}$ and $h_{l^{\prime}, m^{\prime},(l, m)}^{n-2}$. Their sum is $\mathbf{0}$ by $(22)$ applied to them all. Again the norm of the difference between a partial sum and 1 tends to 0 .

Again it is easy to check that the convergence occurs not only in the $L_{1}$ norm, but also in any $L_{p}$ norm for $p<\infty$, in the same way as in Remark 1 - on each of the cubes the $L_{\infty}$ norm of the partial sums is bounded by 1 .

One may wonder why the same arguments do not imply the convergence of the series arranged by rows in $G_{n}$ and columns in $H_{n-1}$ to $(\mathbf{0}, \mathbf{1})$. The answer is that we lack the equivalent of property (22) for this arrangement. To illustrate this let us look at the 3-Kadets family given at the beginning of the section arranged in this natural way. The $\operatorname{sum} \sum_{j=1}^{n+1} g_{m, j}^{n}$ on $Q_{2}$ is equal to 1 on $(m-1) / n<u_{n}<m / n$, while the sum of the appropriate column of $H_{n-1}, \sum_{j=1}^{n+1} \sum_{m^{\prime}=1}^{n-1} \sum_{j^{\prime}=1}^{n} h_{m^{\prime}, j^{\prime},(m-1)(n+1)+j}^{n-1}$, is equal to $-1 / n$ on the whole cube $Q_{2}$. Thus the partial sums before each function of the first kind do not vanish as they did in the previous three cases, and when half of these functions from a given $F_{n}$ have appeared, the norm of the partial sum on $Q_{2}$ is $1 / 2$ regardless of $n$-thus this particular series does not converge. Of course we still have to prove this is true for any rearrangement; but this example indicates why only three and not four possible limits exist.

4. Auxiliary lemmas. Before we begin the main part of this paper, i.e. the proof that our series cannot converge to $(\mathbf{0}, \mathbf{1})$, we shall need three auxiliary lemmas:

Lemma 1 (given without proof in [O89]). Let $(X, \mu)$ and $(Y, \nu)$ be probability measure spaces. Let $f(x, y)$ and $g(x, y)$ be functions in $L_{1}(X \times Y)$, each of which depends on only one variable: $f(x, y)=\tilde{f}(x), g(x, y)=\tilde{g}(y)$. Then

$$
\|f+g\| \geq\|f\|+\|g\|[1-2 \mu(\operatorname{supp} \tilde{f})] .
$$


Proof. We have

$$
\begin{aligned}
\|f+g\| & =\int_{X \times Y}|f+g|=\int_{\operatorname{supp} \tilde{f} \times Y}|f+g|+\int_{(X \backslash \operatorname{supp} \tilde{f}) \times Y}|g| \\
& \geq \int_{\operatorname{supp}(\tilde{f}) \times Y}|f|-\int_{\operatorname{supp}(\tilde{f}) \times Y}|g|+(1-\mu(\operatorname{supp} \tilde{f}))\|g\| \\
& =\|f\|-\mu(\operatorname{supp} \tilde{f})\|g\|+(1-\mu(\operatorname{supp} \tilde{f}))\|g\| \\
& =\|f\|+\|g\|[1-2 \mu(\operatorname{supp} \tilde{f})] .
\end{aligned}
$$

Lemma 2. Let $A, B, C$ be probability measure spaces and let $X=A \times B \times C$ be equipped with the standard product measure. Suppose $f, g$ are bounded functions defined on $X$ of the form $f(a, b, c)=\tilde{f}(a, b)=\sum_{k=1}^{N} s_{k} \chi_{A_{k} \times B_{k}}$ and $g(a, b, c)=\tilde{g}(b, c)=\sum_{l=1}^{N} t_{l} \chi_{B_{l} \times C_{l}}$, and $\|f-g\| \leq \varepsilon$. Then there exists $a$ function $h(a, b, c)=\tilde{h}(b)$ such that $\|h-g\| \leq 2 \varepsilon$ and $\|h-f\| \leq 2 \varepsilon$. Moreover if $f$ is integer-valued then $h$ can also be chosen to be integer-valued, and if for a family of sets $B_{\alpha} \subset B$ we have $\forall_{\alpha} \forall_{b_{1}, b_{2} \in B_{\alpha}} \forall_{a \in A} f\left(a, b_{1}, c\right)=f\left(a, b_{2}, c\right)$, then we can choose $h$ constant on each $B_{\alpha}$.

Proof. For any given $b \in B$ we take $\tilde{h}(b)$ such that

$$
\int_{A}|\tilde{f}(a, b)-\tilde{h}(b)| d a=\inf _{x \in \mathbb{R}}\left\{\int_{A}|\tilde{f}(a, b)-x| d a\right\} .
$$

This is well defined, as $f$ is bounded, and thus in fact the inf is taken over a bounded, and thus compact set. For such an $h$ we have

$$
\begin{aligned}
\|h-f\| & =\int_{X}|f(a, b, c)-\tilde{h}(b)|=\iint_{C} \int_{B}|\tilde{f}(a, b)-\tilde{h}(b)| \\
& =\int_{C} \int_{B} \inf \left\{\int_{A}|\tilde{f}(a, b)-x(b)|\right\} \leq \iint_{C} \int_{A}|\tilde{f}(a, b)-\tilde{g}(b, c)| \\
& \leq \int_{C} \int_{B} \int_{A}|f(a, b, c)-g(a, b, c)|=\|f-g\| \leq \varepsilon .
\end{aligned}
$$

As $\|h-f\| \leq \varepsilon$ and $\|f-g\| \leq \varepsilon$, we immediately have $\|g-h\| \leq 2 \varepsilon$. As for the additional assumptions, if $f$ and $g$ are integer-valued, we can take the inf in the definition of $\tilde{h}$ to be only over the integers, with the same result. Regardless of which option we choose, if $f$ is constant with respect to $b$ on any $B_{\alpha}$, then from the definition $h$ can also be chosen to be constant on that set.

Lemma 3. Let $A, B$ be probability measures and $X=A \times B$ equipped with the standard product measure. Suppose $f, g, h$ are integer-valued functions defined on $X$ with $f(a, b)=\tilde{f}(a)$ and $h(a, b)=\tilde{h}(b)$ for some $\tilde{f}, \tilde{h}$. Suppose also that $g$ assumes only two adjacent values (i.e. $k$ and $k+1$ for some $k$ ). Finally, suppose that $\|f+g+h\|<\delta<1 / 9$. Then either $f$ or $h$ is a constant 
function equal to some integer $c$ on a set of measure $\geq 1-2 \sqrt{\delta}$. Furthermore, $\|f-c\|<3 \sqrt{\delta}$ (or $\|h-c\|<3 \sqrt{\delta}$, respectively).

Proof. The sets $F_{n}=\tilde{f}^{-1}((-\infty, n])$ and $H_{n}=\tilde{h}^{-1}((-\infty, n])$ form two increasing families, the sum of each is the whole space ( $A$ or $B$ respectively) and the intersection of each is empty. The measures $\left|F_{n}\right|$ thus form an ascending sequence with elements arbitrarily close to 0 when $n \rightarrow-\infty$ and arbitrarily close to 1 when $n \rightarrow \infty$. As $F_{n} \backslash F_{n-1}=\tilde{f}^{-1}(n)$, if $\tilde{f}$ is not constant on any set of measure $\geq 1-2 \sqrt{\delta}$, then at least one element of the sequence $\left|F_{n}\right|$, say $\left|F_{n_{f}}\right|$, has to fall into the interval $[\sqrt{\delta}, 1-\sqrt{\delta}]$. Similarly if $\tilde{h}$ is constant on no set of measure $\geq 1-2 \sqrt{\delta}$, then for some $n_{h}$ we have $\sqrt{\delta} \geq\left|H_{n_{h}}\right| \geq 1-\sqrt{\delta}$. Then on the set $X_{1}=F_{n_{f}} \times H_{n_{h}}$ we have $f(a, b)+h(a, b) \leq n_{h}+n_{f}$, while on $X_{2}=\left(A \backslash F_{n_{f}}\right) \times\left(B \backslash H_{n_{h}}\right)$ we have $f(a, b)+h(a, b) \geq n_{h}+n_{f}+2$. As $g$ assumes two adjacent values, it is either $\leq-\left(n_{h}+n_{f}+1\right)$ or $\geq-\left(n_{h}+n_{f}+1\right)$ on the whole $X$. Thus on one of the sets $X_{1}, X_{2}$ (call it $X_{i}$ ) we have $|f+g+h| \geq 1$. As both $X_{1}$ and $X_{2}$ are products of two sets of measure $\geq \sqrt{\delta}$, we have

$$
\begin{aligned}
\|f+g+h\| & =\int_{X}|f(a, b)+g(a, b)+h(a, b)| \geq \int_{X_{i}}|f(a, b)+g(a, b)+h(a, b)| \\
& \geq\left|X_{i}\right| \geq \delta
\end{aligned}
$$

which contradicts the assumptions of the lemma.

Thus one of the functions has to be constant on a large set. Without loss of generality we may assume it is $h$, and that it is equal to some integer $c$. Let us examine the function $f$, taking into account that all the functions are integer-valued, and thus if their sum is non-zero, it is at least 1 :

$$
\begin{aligned}
\delta & >\|f+g+h\| \geq\|f+g+c\|_{A \times \tilde{h}^{-1}(c)} \\
& \geq\left|\{\tilde{f}(a) \notin\{-k-c,-k-c-1\}\} \times \tilde{h}^{-1}(c)\right| \\
& =|\{\tilde{f}(a) \notin\{-k-c,-k-c-1\}\}| \cdot(1-2 \sqrt{\delta}),
\end{aligned}
$$

which implies $\tilde{f}(a) \in\{-k-c,-k-c-1\}$ on a set of measure at least $1-\delta /(1-2 \sqrt{\delta})$. Denote this set by $A^{\prime}$. Now we return to the function $h$ :

$$
\|h-c\|_{X} \leq \frac{1}{1-2 \sqrt{\delta}}\|h-c\|_{A^{\prime} \times B}=\frac{1}{1-2 \sqrt{\delta}}\|h-c\|_{A^{\prime} \times\left(B \backslash \tilde{h}^{-1}(c)\right)} .
$$

On $A^{\prime}$ the function $f+g+c$ assumes values of absolute value $\leq 1$, so by substituting $f+g$ for $-c$ we shall decrease the norm at most by

$$
1 \cdot\left|A^{\prime} \times\left(B \backslash \tilde{h}^{-1}(c)\right)\right| \leq\left(1-\frac{\delta}{1-2 \sqrt{\delta}}\right)(2 \sqrt{\delta}) \leq 2 \sqrt{\delta},
$$


thus obtaining the inequality

$$
\begin{aligned}
\|h-c\|_{X} & \leq \frac{1}{1-2 \sqrt{\delta}}\|h+f+g\|_{A^{\prime} \times\left(B \backslash \tilde{h}^{-1}(c)\right)}+2 \sqrt{\delta} \\
& \leq \frac{1}{1-2 \sqrt{\delta}}\|f+g+h\|_{X}+2 \sqrt{\delta} \leq \frac{\delta}{1-2 \sqrt{\delta}}+2 \sqrt{\delta} .
\end{aligned}
$$

As $\delta \leq 1 / 9$, we have $\delta /(1-2 \sqrt{\delta}) \leq \sqrt{\delta}$, and thus $\|h-c\| \leq 3 \sqrt{\delta}$.

5. The fourth point. Now we can prove the main theorem of the paper:

THEOREM 5.1. The function $d_{\infty}=(\mathbf{0}, \mathbf{1})$ does not belong to the sum range of any 3-Kadets family series.

Proof. Suppose we have a rearrangement of some 3-Kadets family $d_{\sigma(n)}$ whose sum converges to $d_{\infty}$. Again, take a small $\delta>0$ (we shall need $927 \sqrt{\delta}<1 / 4$, i.e. $\delta<1 / 13749264)$ and an integer $K$ satisfying inequalities (15) and (16), i.e. the tails and Cauchy sums are smaller than $\delta$ for $N>K$. Then, again, we take any $M$ satisfying (17), i.e. such that $V_{M}$ contains the first $K$ elements of our series. Then we take an $N_{0}$ such that

$$
V_{M} \subset\left\{d_{\sigma(1)}, \ldots, d_{\sigma\left(N_{0}\right)}\right\} \text {. }
$$

Consider any fixed $N>N_{0}$. We will prove that

$$
\int_{Q_{3}} \sum_{n=1}^{N} d_{\sigma(n)}<\frac{1}{4} .
$$

Clearly this suffices to prove that our series does not converge to 1 on $Q_{3}$, which contradicts the assumption the rearrangement converged to $(\mathbf{0 , 1})$.

For any $L, k \in \mathbb{Z}$ denote by $D_{k}$ the set $\left\{d_{\sigma(1)}, \ldots, d_{\sigma(k)}\right\}$, and by $F_{L}^{k}, G_{L}^{k}$, $H_{L}^{k}$ and $V_{L}^{k}$ the intersections of $F_{L}, G_{L}, H_{L}$ or $V_{L}$, respectively, with $D_{k}$. First we shall prove the following lemma:

LEMMA 4. If functions $f_{m}^{n}, g_{m, j}^{n}$ and $h_{m, j, k}^{n}$ are a 3-Kadets family on the cubes $Q_{1}, Q_{2}$ and $Q_{3}$ and their fixed permutation $d_{\sigma(n)}$ has the property that $\sum d_{\sigma(n)}$ is 0 on $Q_{1}$ and 1 on $Q_{2}$ and $Q_{3}$, and for a given $L$ we have $\int_{Q_{3}} \tilde{G}_{L}^{N} \geq 1 / 2+38 \delta$, where $N>N_{0}$ as above, then there exists a $P \subset[0,1]$ such that $|P|=1 / 2$ and $\left[\left(\tilde{H}_{L}^{N}\right)^{-1}(0)\right] \cap\left\{v: v_{L} \in P\right\} \subset Q_{3}$ has measure $\leq 450 \delta$.

REMARK 2. What this lemma really tells us is this: if up to the $N$ th element of the series at least half plus something $(38 \delta)$ of the $G_{L}$ functions have appeared, then at least half minus something $(450 \delta)$ of the $H_{L}$ functions had to appear. Moreover, the $H_{L}$ functions do not appear in a haphazard fashion - we know that at least half minus something rows had to appear (a row is the set of all functions $h_{m, j, k}^{L}$ with fixed $m$ and $j$ and varying $k$ ). 
Proof of Lemma 4. If $L \leq M$ then our assertion is automatically satisfied -all functions from $H_{L}$ belong to $D_{N}$, thus we can take any set of measure $1 / 2$ for $P$ and the set $\left(\tilde{H}_{L}^{N}\right)^{-1}(0)$ will be empty, so $P$ will satisfy the required conditions.

Now consider the case $L>M$. The numbers $K$ and $L-1$ satisfy conditions (15)-(17) (as $L>M$ and $M$ satisfies (17)). Thus there exist numbers $n_{i}$ satisfying (18) (with $n_{i}$ in place of $k_{i}$ ). We shall prove that $N \geq n_{2}$.

We know that $\int_{Q_{3}} \tilde{G}_{L}^{N}=-\int_{Q_{1}} \tilde{G}_{L}^{N}$ (as all $g_{m, j}^{n}$ are of the same constant sign on each cube, the absolute value of the integral is equal to the norm, and the norms on each cube are equal). If $N<n_{2}$, then

$$
\left\|\tilde{G}_{L}^{N}\right\|_{Q_{1}} \leq\left\|\tilde{G}_{L}^{n_{2}}\right\|_{Q_{1}}=\left\|\overline{\bar{d}}_{1}+\overline{\bar{d}}_{2}\right\| \leq\left\|d_{1}^{* *}\right\|+19 \delta+\left\|d_{2}^{* *}\right\|+19 \delta<\frac{1}{2}+38 \delta,
$$

which contradicts our assumption (the first inequality follows from the fact that $g_{m, j}^{n}$ are non-positive functions on $Q_{1}$, and the second inequality from Corollary 2.4).

Thus $N>n_{2}$. Consider $\tilde{V}_{L-1}^{n_{2}}+\tilde{F}_{L}^{n_{2}}$ on $Q_{1}$. This function depends on $t_{1}, \ldots, t_{L}$, while $\tilde{G}_{L}^{n_{2}}=\overline{\bar{d}}_{1}+\overline{\bar{d}}_{2}$ on $Q_{1}$ depends on $t_{L}$ and $t_{L+1}$. From property (15) and Corollary 2.4 we get

$$
\left\|\tilde{V}_{L-1}^{n_{2}}+\tilde{F}_{L}^{n_{2}}+\tilde{G}_{L}^{n_{2}}\right\| \leq\left\|\tilde{D}_{k}\right\|+\left\|d_{1}^{* *}+\overline{\bar{d}}_{1}\right\|+\left\|d_{2}^{* *}+\overline{\bar{d}}_{2}\right\| \leq \delta+19 \delta+19 \delta=39 \delta .
$$

We can thus use Lemma 2 to show that on $Q_{1}$ both functions $-\tilde{V}_{L-1}^{n_{2}}-\tilde{F}_{L}^{n_{2}}$ and $\tilde{G}_{L}^{n_{2}}$ are both closer than $39 \delta$ to some integer-valued function $\tilde{A}$ depending only on $t_{L}$.

Each function $f_{m}^{n}$ depends only on $t_{n}$ and assumes the values 0 and 1 only (properties (5) and (4)), so it is in fact the characteristic function of a set $\left\{t: t_{n} \in S_{m}^{n}\right\}$ for some $S_{m}^{n} \subset[0,1]$. As the $f_{m}^{n}$ have disjoint supports for fixed $n$, they are all constant on any given $S_{m}^{n}$. The $g$ functions are also constant with respect to $t_{n}$ on $S_{m}^{n}$ by (6), and all the other functions are constant with respect to $t_{n}$ on the whole interval. Thus the functions $-\tilde{V}_{L-1}^{n_{2}}-\tilde{F}_{L}^{n_{2}}$ and $\tilde{G}_{L}^{n_{2}}$ are constant with respect to $t_{L}$ on $\left\{t_{L} \in S_{m}^{L}\right\}$, so we can choose $\tilde{A}$ to be constant on those sets. Thus $\tilde{A}$ coincides on $Q_{1}$ with the sum of some of the rows of $G_{L}$, i.e. $\tilde{A}$ corresponds to some subset $A$ of $G_{L}$ such that for fixed $m$ either all or none of the functions $g_{m, j}^{L}$ belong to $A$. Define $\tilde{A}$ on $Q_{2}$ and $Q_{3}$ as the sum of all the elements of $A$ as well, which agrees with our notation that $\tilde{U}$ is the sum of all the elements of $U$ for an arbitrary set of functions.

We know from (18) and Proposition 2.5 that

$$
\left\|\sum_{n=n_{2}+1}^{\infty} d_{n}^{*}\right\|_{Q_{1}} \leq \frac{1-\delta}{4}+\frac{1-\delta}{4}+11 \delta \leq \frac{1}{2}+11 \delta .
$$


Note that $\left.\left(\tilde{V}_{L-1}^{n_{2}}+\tilde{F}_{L}^{n_{2}}+\sum_{n=n_{2}+1}^{\infty} d_{n}^{*}\right)\right|_{Q_{1}}=\left.\left(\tilde{V}_{L-1}+F_{L}\right)\right|_{Q_{1}}=\left.\mathbf{1}\right|_{Q_{1}}$, so $\left\|\tilde{V}_{L-1}^{n_{2}}+\tilde{F}_{L}^{n_{2}}\right\|_{Q_{1}} \geq 1 / 2-11 \delta$. On the other hand,

$$
\left\|\tilde{V}_{L-1}^{n_{2}}+\tilde{F}_{L}^{n_{2}}\right\|_{Q_{1}}=\left\|\tilde{D}_{K}+d_{1}^{* *}+d_{2}^{* *}\right\|_{Q_{1}} \leq \delta+\frac{1-\delta}{4}+\frac{1-\delta}{4} \leq 1 / 2+\delta .
$$

As $\left\|\tilde{V}_{L-1}^{n_{2}}+\tilde{F}_{L}^{n_{2}}-\tilde{A}\right\|_{Q_{1}} \leq 39 \delta$, taking into account the equality $\|\tilde{A}\|_{Q_{1}}=$ $\|\tilde{A}\|_{Q_{2}}$ we get the estimate

$$
\frac{1}{2}-50 \delta \leq\|\tilde{A}\|_{Q_{2}} \leq \frac{1}{2}+40 \delta .
$$

Distinct functions from $G_{L}$ have disjoint supports on $Q_{1}$ (this follows from properties (13) and (6) of Kadets families), and each has the same norm $\psi=1 /\left|M_{L} \times J_{L}\right|$. Thus if the distance between two functions corresponding to two subsets of $G_{L}$ on $Q_{1}$ is smaller than $n \psi$, then at most $n$ functions belong to the symmetric difference of those two subsets. Therefore the distance between the two functions on $Q_{2}$ is at most $n \psi$ (as on $Q_{2}$ the norm of a single function is also $\psi$ by (26)). Thus, in general, if $B, C \subset G_{L}$, then $\|\tilde{B}-\tilde{C}\|_{Q_{1}} \geq\|\tilde{B}-\tilde{C}\|_{Q_{2}}$. In particular $\tilde{G}_{L}^{n_{2}}$ is at most $39 \delta$ distant from $\tilde{A}$ on $Q_{2}$.

Now consider what happens on $Q_{2}$. From (23) the restriction of $\tilde{A}$ to $Q_{2}$ is equal to 1 on some set (on the intervals $t_{L} \in[(m-1) / L, m / L]$ for $m$ such that $g_{m, j}^{L} \in A$ ) and 0 on the rest. From (15), as $n_{2}>K$, we have $\left\|\tilde{D}_{n_{2}}-1\right\|_{Q_{2}} \leq \delta$. If we substitute $\tilde{A}$ for $\tilde{G}_{L}^{n_{2}}$, we will be at most $40 \delta$ distant from zero, precisely

$$
\left\|\tilde{D}_{n_{2}}-1-\tilde{G}_{L}^{n_{2}}+\tilde{A}\right\|_{Q_{2}} \leq 40 \delta .
$$

However, as only $G_{L}$ and $H_{L}$ depend on $u_{L}$, this sum is composed of two parts: the part $\tilde{A}+\tilde{H}_{L}^{n_{2}}$ depending on $u_{L}$ and the rest (i.e. $\left.\tilde{D}_{n_{2}}-\left(\tilde{G}_{L}^{n_{2}}+\tilde{H}_{L}^{n_{2}}\right)\right)$ depending on other variables. Thus we can apply a simplified version of Lemma 2, with $f=\tilde{A}+\tilde{H}_{L}^{n_{2}}, g=-\left(\tilde{D}_{n_{2}}-V_{L}^{n_{2}}\right)$, and a trivial one-point space as $B$. We learn that both our functions are within $80 \delta$ of a function $c$ depending on $b$ (the variable on $B$, as in Lemma 2 ). But as $B$ is a one-point space, $c$ is a constant function. As $\tilde{A}$ assumes the values 0 and 1 , and $\tilde{H}_{L}^{n_{2}} \in[-1,0]$, their sum is non-negative on $\operatorname{supp} \tilde{A}$ and non-positive on the remainder of $Q_{2}$.

From (30) we know that $|\operatorname{supp} \tilde{A}| \geq 1 / 2-50 \delta$, thus $\tilde{A}+\tilde{H}_{L}^{n_{2}}$ is non-negative on a set of measure $\geq 1 / 2-50 \delta$. If $c$ is positive, then (as $\delta<1 / 200$ )

$$
80 \delta \geq\left\|\tilde{A}+\tilde{H}_{L}^{n_{2}}-c\right\| \geq c(1 / 2-50 \delta) \geq \frac{c}{4},
$$

which implies $c \leq 320 \delta$. Similarly if $c$ is negative, we know from (30) that $\left|Q_{2} \backslash \operatorname{supp} \tilde{A}\right| \geq 1 / 2-40 \delta$, yielding again $c>-800 \delta / 3$. Thus $|c|<320 \delta$, so $\left\|\tilde{A}+\tilde{H}_{L}^{n_{2}}\right\| \leq\left\|\tilde{A}+\tilde{H}_{L}^{n_{2}}-c\right\|+|c| \leq 80 \delta+320 \delta=400 \delta$. 
Thus $\tilde{H}_{L}^{n_{2}}$ is within $400 \delta$ of a function with values 0 and -1 on $Q_{2}$, namely $-\tilde{A}$. Note that $-\tilde{A}=-\tilde{A}^{\prime}$ on $Q_{2}$ for a subset $A^{\prime}$ of $H_{L}$ with the property that for given $m$ either all $h_{m, j, k}^{L}$ belong to $A^{\prime}$, or none does (if a given $g_{m, j}^{L}$ belongs to $A$, then all $h_{m, j, k}^{L}$ belong to $A^{\prime}$ ). If $\tilde{A}^{\prime}$, where $A^{\prime} \subset H_{L}$, assumes only the values 0 and 1 on $Q_{2}$ and $B \subset H_{L}$, then

$$
\begin{aligned}
\left\|\tilde{A}^{\prime}-\tilde{B}\right\|_{Q_{2}}= & \left\|\tilde{A}^{\prime}-\tilde{B}\right\|_{\text {supp } \tilde{A}^{\prime}}+\left\|\tilde{A}^{\prime}-\tilde{B}\right\|_{\mathbb{Q}_{2} \backslash \operatorname{supp} \tilde{A}^{\prime}} \\
= & \frac{1}{\left|M_{L} \times J_{L} \times K_{L}\right|}\left|\left\{h: h \in A^{\prime} \wedge h \notin B\right\}\right| \\
& +\frac{1}{\left|M_{L} \times J_{L} \times K_{L}\right|}\left|\left\{h: h \notin A^{\prime} \wedge h \in B\right\}\right| \\
= & \frac{1}{\left|M_{L} \times J_{L} \times K_{L}\right|}|A \triangle B|=\| \tilde{A}^{\prime}-\left.\tilde{B}\right|_{Q_{3}} .
\end{aligned}
$$

Take any subset $A^{\prime \prime}$ of $H_{L}$ depending only on $m$ and $j$ with exactly half of the elements of $H_{L}$ and containing $A^{\prime}$ or contained in $A^{\prime}$. If $B \subset C \subset H_{L}$ or $C \subset B \subset H_{L}$, then $\|\tilde{C}-\tilde{B}\|=|\|\tilde{C}\|-\|\tilde{B}\||$, because all the functions in $H_{L}$ are non-positive. As $\tilde{A}^{\prime}=-\tilde{A}$ on $Q_{2}$ and $\left|\|\tilde{A}\|_{Q_{2}}-1 / 2\right| \leq 50 \delta$ from (30), we get $\left\|\tilde{A}^{\prime}-\tilde{A^{\prime \prime}}\right\|_{Q_{2}} \leq 50 \delta$, and thus $\left\|H_{L}^{n_{2}}-\tilde{A}^{\prime \prime}\right\|_{Q_{3}}=\left\|H_{L}^{n_{2}}-\tilde{A}^{\prime \prime}\right\|_{Q_{2}} \leq 450 \delta$.

Now consider what happens on $Q_{3}$. As $\tilde{H}_{L}^{n_{2}}$ and $\tilde{A}^{\prime \prime}$ are both integervalued on $Q_{3}$, they differ on a set of measure at most $450 \delta$, and thus their difference can be positive on a set of measure at most $450 \delta$. When we increase $n$ from $n_{2}$ to $N$ the set where the difference is positive can only decrease. Thus $\left|\left\{H_{L}^{N}-\tilde{A}^{\prime \prime}>0\right\}\right| \leq 450 \delta$. Now we take supp $\tilde{A}^{\prime \prime}$ for $P$. Then $\left[\left(\tilde{H}_{N}^{L}\right)^{-1}(0)\right] \cap\left\{v: v_{L} \in P\right\}$ is the set where $H_{N}^{L}$ is zero and $\tilde{A}^{\prime \prime}$ is negative, thus their difference is positive, so the set has measure smaller than $450 \delta$, which is what we had to prove.

Now the main proof. Assume $d_{\infty}=(\mathbf{0}, \mathbf{1})$, i.e. our series converges to $\mathbf{1}$ on $Q_{2}$ and $Q_{3}$ and to $\mathbf{0}$ on $Q_{1}$. We shall prove by induction upon $L$ that

$$
\int_{Q_{3}} \tilde{V}_{L}^{N} \leq \frac{1}{4} .
$$

As $\sum_{n=1}^{N} d_{\sigma(n)}$ is finite, its elements are contained in some $V_{L}$, thus if our statement is true, we get $\int_{Q_{3}} \sum_{n=1}^{N} d_{\sigma(n)} \leq 1 / 4$, which is what we had to prove. For $L<M$ we have $V_{L} \subset D_{N}$ and $\int_{Q_{3}} \tilde{V}_{L}^{N}=0 \leq 1 / 4$ from (7).

Now suppose we have the statement for $L-1$. Set

$$
P_{1}=\left.\left(\tilde{V}_{L-1}^{N}+\tilde{G}_{L}^{N}\right)\right|_{Q_{3}}, \quad P_{2}=\sum_{n>L}\left(\tilde{G}_{n}^{N}+\left.\tilde{H}_{n}^{N}\right|_{Q_{3}}\right) .
$$

Consider the function $\left.\tilde{H}_{L}^{N}\right|_{Q_{3}}$. It depends on the variables $v_{L}$ and $v_{L+1}$. The function $P_{1}$ depends on $v_{1}, \ldots, v_{L}$, while $P_{2}$ depends on $v_{L+1}, \ldots, v_{Z}$ for some 
$Z \in \mathbb{Z}$. The function $\left.H_{L}^{N}\right|_{Q_{3}}$ assumes only the values 0 and -1 , all three functions $-\left.H_{L}^{N}\right|_{Q_{3}}, P_{1}$ and $P_{2}$ are integer-valued, and from (15) their sum is less than $\delta$ distant from 1 on $Q_{3}$. Thus by taking $P_{1}^{\prime}=P_{1}-1$ we have three functions satisfying the assumptions of Lemma 3 . Thus either $P_{1}$ or $P_{2}$ is within $3 \sqrt{\delta}$ of a constant function. In each of these cases the proof will also depend on whether $\int_{Q_{3}} \tilde{G}_{L}^{N} \leq 1 / 2+38 \delta$ or $\int_{Q_{3}} \tilde{G}_{L}^{N}>1 / 2+38 \delta$. Thus we have in total four cases to consider.

Suppose first that $P_{2}$ is within $3 \sqrt{\delta}$ of a constant function. As $\| P_{1}+$ $P_{2}+\tilde{H}_{L}^{N}-1 \| \leq \delta$, this means that $P_{1}+\tilde{H}_{L}^{N}$ is within $3 \sqrt{\delta}+\delta \leq 4 \sqrt{\delta}$ of a constant function. If $\int_{Q_{3}} \tilde{G}_{L}^{N} \leq 1 / 2+38 \delta$, then

$$
\int_{Q_{3}} \tilde{V}_{L}^{N}=\int_{Q_{3}}\left(\tilde{V}_{L-1}^{N}+\tilde{G}_{L}^{N}+\tilde{H}_{L}^{N}\right) \leq \frac{1}{4}+\left(\frac{1}{2}+38 \delta\right)+0=\frac{3}{4}+38 \delta .
$$

But $\tilde{V}_{L}^{N}$ is equal to $P_{1}+\tilde{H}_{L}^{N}$, and so is within $4 \sqrt{\delta}$ of some constant integer $c$ and its integral also has to be within $4 \sqrt{\delta}$ of $c$. As $4 \sqrt{\delta}+38 \delta<1 / 4$, we get $c \leq 0$, thus $\int_{Q_{3}} \tilde{V}_{L}^{N} \leq c+4 \sqrt{\delta} \leq 1 / 4$.

If $P_{2}$ is within $3 \sqrt{\delta}$ of a constant function, and $\int_{Q_{3}} \tilde{G}_{L}^{N}>1 / 2+38 \delta$, then again $P_{1}+\tilde{H}_{L}^{N}$ is within $4 \sqrt{\delta}$ of a constant integer $c$. From Lemma 4 we deduce in particular that $\int_{Q_{3}} \tilde{H}_{L}^{N} \leq-1 / 2+450 \delta$. Obviously $\int_{Q_{3}} \tilde{G}_{L}^{N} \leq 1$, thus

$$
\int_{Q_{3}} V_{L}^{N}=\int_{Q_{3}}\left(V_{L-1}^{N}+\tilde{G}_{L}^{N}+\tilde{H}_{L}^{N}\right) \leq \frac{1}{4}+1-\frac{1}{2}+450 \delta=\frac{3}{4}+450 \delta .
$$

As $\int_{Q_{3}} V_{L}^{N}$ is supposed again to be within $4 \sqrt{\delta}$ of $c$, we have $c \leq 0$ as $450 \delta+4 \sqrt{\delta} \leq 1 / 4$. Thus again $\int_{Q_{3}} \tilde{V}_{L}^{N} \leq c+4 \sqrt{\delta} \leq 1 / 4$.

In the third case we suppose that $P_{1}^{\prime}$, and thus also $P_{1}$, is within $3 \sqrt{\delta}$ of a constant function, and $\int_{Q_{3}} \tilde{G}_{L}^{N} \leq 1 / 2+38 \delta$. As $\int_{Q_{3}} \tilde{V}_{L-1}^{N} \leq 1 / 4$ from the inductive assumption, we have $\int_{Q_{3}} P_{1} \leq 3 / 4+38 \delta$. As $P_{1}$ is supposed to be within $3 \sqrt{\delta}$ of some constant integer $c$, its integral also has to be within $3 \sqrt{\delta}$ of $c$, which again implies $c \leq 0$ and $\int_{Q_{3}} P_{1} \leq 3 \sqrt{\delta}$. As $\tilde{V}_{L}^{N}=P_{1}+\tilde{H}_{L}^{N}$ and $\tilde{H}_{L}^{N} \leq 0$, we get $\int_{Q_{3}} \tilde{V}_{L}^{N} \leq 3 \sqrt{\delta} \leq 1 / 4$.

The last case is when $P_{1}$ is within $3 \sqrt{\delta}$ of a constant integer $c$ and $\int_{Q_{3}} \tilde{G}_{L}^{N}>$ $1 / 2+38 \delta$. In this case from Lemma 4 we know there exists a set $P^{\prime} \subset Q_{3}$ depending only on $v_{L}$ such that $\left|P^{\prime}\right|=1 / 2$ and $\int_{P^{\prime}} \tilde{H}_{L}^{N} \leq-1 / 2+450 \delta$. If $P_{1}$ is within $3 \sqrt{\delta}$ of a constant integer function and $P_{1}+P_{2}+\tilde{H}_{L}^{N}$ is within $\delta$ of 1 (from (15)) then $P_{2}+\tilde{H}_{L}^{N}$ is within $3 \sqrt{\delta}+\delta \leq 4 \sqrt{\delta}$ of some constant integer function $C$. Taking $P_{2}^{\prime}=P_{2}-C$ we arrrive at the situation of Lemma 2: $\tilde{H}_{L}^{N}$ depends on $v_{L}$ and $v_{L+1}$ while $P_{2}^{\prime}$ depends on $v_{L+1}, v_{L+2}, \ldots, v_{Z}$. This means that each of them is within $8 \sqrt{\delta}$ of some integer function $P_{3}$ depending only 
on $v_{L+1}$. As $\int_{P^{\prime}} \tilde{H}_{L}^{N} \leq-1 / 2+450 \delta$ and $\left\|\tilde{H}_{L}^{N}-P_{3}\right\| \leq 8 \sqrt{\delta}$, we gather that

$$
\int_{P^{\prime}} P_{3} \leq-\frac{1}{2}+450 \delta+8 \sqrt{\delta} \leq-\frac{1}{2}+458 \sqrt{\delta} .
$$

As $P^{\prime}$ depends only on $v_{L}$ and $P_{3}$ only on $v_{L+1}$ and $\left|P^{\prime}\right|=\left|Q_{3} \backslash P^{\prime}\right|$,

$$
\int_{Q_{3}} P_{3}=\int_{P^{\prime}} P_{3}+\int_{Q_{3} \backslash P^{\prime}} P_{3}=2 \int_{P^{\prime}} P_{3} \leq-1+916 \sqrt{\delta} .
$$

Returning to $\tilde{H}_{L}^{N}$ we get $\int_{Q_{3}} \tilde{H}_{L}^{N} \leq \int_{Q_{3}} P_{3}+8 \sqrt{\delta} \leq-1+924 \sqrt{\delta}$.

As $\int_{Q_{3}} \tilde{G}_{L}^{N} \leq 1$ and $\int_{Q_{3}} \tilde{V}_{L-1}^{N} \leq 1 / 4$ we get $\int_{Q_{3}} P_{1} \leq 5 / 4$. As before, $\int_{Q_{3}} P_{1}$ has to be within $3 \sqrt{\delta}$ of the integer $c$, implying $c \leq 1$ and $\int_{Q_{3}} P_{1} \leq 1+3 \sqrt{\delta}$. We have

$$
\int_{Q_{3}} \tilde{V}_{L}^{N}=\int_{Q_{3}}\left(P_{1}+\tilde{H}_{L}^{N}\right) \leq 1+3 \sqrt{\delta}-1+924 \sqrt{\delta} \leq 927 \sqrt{\delta} \leq \frac{1}{4} .
$$

Thus in all four cases we have completed the induction step, which proves in a finite number of steps that $\int_{Q_{3}} \tilde{D}_{N} \leq 1 / 4$. This holds for an arbitrary $N>N_{0}$, and would thus have to hold for the limit function, $\int_{Q_{3}} d_{\infty} \leq 1 / 4$, which obviously contradicts the assumption that $\left.d_{\infty}\right|_{Q_{3}}=\mathbf{1}$.

Corollary 5.2. A 3-Kadets series has a 3-point sum range, consisting of the functions $(\mathbf{0}, \mathbf{0}),(\mathbf{1}, \mathbf{0})$ and $(\mathbf{1}, \mathbf{1})$. As previously, this holds for any $L_{p}$ with $1 \leq p<\infty$.

6. More points. From the previous section we know how to make 3 points out of 2 . The same mechanism can be applied to make $r+1$ points out of $r$.

TheOREM 6.1. For any $r>1$ there exists a family $\left\{d_{k}\right\}$ of functions defined on a union of cubes $Q_{1}, \ldots, Q_{N}$ with an r-point sum range. Additionally we can distinguish two disjoint subsets $\mathcal{F}$ and $\mathcal{G}$ of $\left\{d_{k}: k \in \mathbb{N}\right\}$ which form a Kadets family on $Q_{N}$, while all other functions $d_{k}$ vanish on $Q_{N}$. Moreover one function in the sum range of $d_{k}$ is equal to 1 on $Q_{N}$ and all the other functions from the sum range vanish on $Q_{N}$. Finally, there exist rearrangements convergent to any point of the sum range in which the sets $\mathcal{F}$ and $\mathcal{G}$ are arranged as in Proposition 2.1.

Proof. We shall prove the assertion by induction on $r$. For $r=2$ the original Kadets example with $N=1$ satisfies the given conditions.

Suppose we have an appropriate family for $r-1$. We add two cubes to the domain of $d_{k}: Q_{N+1}$ and $Q_{N+2}$. Denote by $x=\left(x_{1}, x_{2}, \ldots\right)$ the variable on $Q_{N+1}$ and by $y=\left(y_{1}, y_{2}, \ldots\right)$ the variable on $Q_{N+2}$. All the functions not in $\mathcal{G}$ will vanish on these cubes. For each $n$ we divide the unit interval 
$[0,1]$ into $\left|M_{n}\right|$ sets $S_{m}^{n}, m \in M_{n}$, of measure $1 /\left|M_{n}\right|$ each. We define $g_{m, j}^{n}$ to be $1 /\left|J_{n}\right|$ if $x_{n} \in S_{m}^{n}$, and 0 otherwise. Next we define $K_{n}=M_{n+1} \times J_{n+1}$ and divide the unit interval $[0,1]$ into $\left|K_{n}\right|$ sets $T_{k}^{n}$ of equal measure, and on $Q_{N+2}$ define $g_{m, j}^{n}$ to be 1 if $y_{n} \in T_{(m, j)}^{n-1}$, and 0 otherwise. Finally, to the functions $d_{k}$ we add a set of functions $\mathcal{H}=\left\{h_{m, j, k}^{n}\right\}$ which vanish on the cubes $Q_{1}$ to $Q_{N}$, and satisfy

$$
h_{m, j, k}^{n}=-\frac{1}{\left|K_{n}\right|} g_{m, j}^{n} \quad \text { on } Q_{N+1}, \quad h_{m, j, k}^{n}=-g_{m, j}^{n} \cdot g_{k}^{n+1} \quad \text { on } Q_{M+2} .
$$

It is again easy, although tedious, to check that $\mathcal{F}, \mathcal{G}$ and the new functions $\mathcal{H}$ form a 3-Kadets family on $Q_{N}, Q_{N+1}, Q_{N+2}$. We claim that the set $\left\{d_{k}\right\} \cup \mathcal{H}$ satisfies the conditions of the theorem. The sets $\mathcal{G}$ and $\mathcal{H}$ form a Kadets family on $Q_{N+2}$, and all other functions vanish on $Q_{N+2}$. We have to check the sum ranges. Fix any convergent rearrangement $e_{k}$ of $\left\{d_{k}\right\} \cup \mathcal{H}$. From the properties of 3-Kadets families given in Section 3 we know that the limit on $Q_{N+1}$ and $Q_{N+2}$ is the same, and equals either $\mathbf{0}$ or $\mathbf{1}$. From Theorem 5.1 we know that if the series converges to $\mathbf{0}$ on $Q_{M}$, it has to converge to $\mathbf{0}$ on $Q_{N+1}$ and $Q_{N+2}$. Thus at most $r+1$ limits can be achieved: the functions with 0 on $Q_{N}$ generate one each (by the 0-extension onto $Q_{N+1} \cup Q_{N+2}$ ), while the single function with $\mathbf{1}$ on $Q_{N}$ can be extended by either $\mathbf{0}$ or $\mathbf{1}$ to $Q_{N+1} \cup Q_{N+2}$. This also satisfies the condition that only one of the points in the sum range is $\mathbf{1}$ on $Q_{N+2}$, while the others vanish on $Q_{2}$.

We can obviously attain all the desired points in the sum range with $\mathcal{G}$ and $\mathcal{H}$ ordered as in Proposition 2.1 by taking the rearrangements with $\mathcal{F}$ and $\mathcal{G}$ ordered as in the proposition and inserting $\mathcal{H}$ as in Section 3.

Thus it is possible to attain an affine-independent finite set of any size $r$ as the sum range of a conditionally convergent series. Again, this works for any $L_{p}, 1 \leq p<\infty$.

To reach full generality on $L_{p}$ we will attain arbitrary sum ranges, and not only affine-independent ones. The proof presented below is due to the anonymous referee of this paper and follows the scheme from [K90].

Lemma 5. Let $\Omega$ be a probability space, $c_{n} \in \mathbb{R}$ with $c_{n} \rightarrow 0$, and let $f_{n} \in L_{2}(\Omega)$ be a sequence of integer-valued functions. Then the series $\sum_{n=1}^{\infty}\left(f_{n}+c_{n}\right)$ converges if and only if both $\sum_{n=1}^{\infty} f_{n}$ and $\sum_{n=1}^{\infty} c_{n}$ converge.

Proof. The "if" part is obvious. For the "only if" part it is enough to prove that if $\sum c_{n}$ diverges, then $\sum\left(f_{n}+c_{n}\right)$ has to diverge as well. In fact, if $\sum c_{n}$ diverges then there exists an $\varepsilon \in(0,1 / 4)$ such that for any $N \in \mathbb{N}$ we have a large Cauchy sum above $N$, i.e. for some $l>k>N$ we have $\left|\sum_{n=k}^{l} c_{n}\right|>\varepsilon$. As $c_{n} \rightarrow 0$ we can take $N$ large enough to ensure $\left|c_{j}\right|<\varepsilon$ for $j>N$. Thus we can select $l=l(k)$ such that $\varepsilon<\sum_{n=k}^{l(k)} c_{n}<2 \varepsilon<1 / 2$. 
But then $\left\|\sum_{n=k}^{l(k)}\left(f_{n}+c_{n}\right)\right\| \geq \varepsilon$ as a sum of an integer-valued function and a constant $c \in(\varepsilon, 1 / 2)$, which ensures the divergence of $\sum\left(f_{n}+c_{n}\right)$.

Now let us apply this lemma to our example from Theorem 6.1. We have a series $d_{k}$ with an $r+2$-point sum range $D$ defined on the union $\Omega=\bigcup_{i=1}^{2 r+1} Q_{i}$ of cubes. We consider it as a series defined on $L_{2}(\Omega)$. Let $X=\operatorname{lin}\left\{\chi_{Q_{1}}, \ldots, \chi_{Q_{2 r+1}}\right\}$ be the subspace of piecewise constant functions on $\Omega$. Let $P: L_{2}(\Omega) \rightarrow X$ be the orthogonal projection onto $X$. Denote by $Y$ the subspace of $X$ consisting of those piecewise constant functions $\left(f_{i}\right)_{i=1}^{2 r+1}$, where $f_{i}$ is the value of $f$ on $Q_{i}$, that satisfy $f_{2 j}=f_{2 j+1}$ for $j=1, \ldots, r$.

Recall that $\int_{Q_{2} j} d_{k} d \mu=\int_{Q_{2 j+1}} d_{k} d \mu$ for $j=1, \ldots, r$. Thus for any $d_{k}$ we have $P\left(d_{k}\right) \in Y$, and thus $P(D)$ is in fact a subset of $Y$. Recall also that for odd indices $j$ the functions $d_{k}$ are integer-valued. Let $T: Y \rightarrow Y$ be an arbitrary linear operator. Put $d_{k}^{\prime}=d_{k}+T P\left(d_{k}\right)$.

Theorem 6.2. The sum range $D^{\prime}$ of the series $\sum d_{k}^{\prime}$ equals $(I+T)(D)$.

Proof. The inclusion $(I+T)(D) \subset D^{\prime}$ is evident. To prove the inverse inclusion consider an arbitrary arrangement $\left(b_{k}^{\prime}\right)$ of $\left(d_{k}^{\prime}\right)$ and the corresponding rearrangement $\left(b_{k}\right)$ of $\left(d_{k}\right)$. If $\left(b_{k}^{\prime}\right)$ converges to some $b^{\prime} \in D^{\prime}$, then its restrictions to $Q_{j}$ for odd indices $j$ satisfy the conditions of the lemma. Thus the restrictions of $T P\left(b_{k}\right)$ to $Q_{j}$ for odd $j$ converge. Now the restrictions of $T P\left(b_{k}\right)$ to $Q_{j-1}$ are equal to the corresponding restrictions to $Q_{j}$, so the whole series $T P\left(b_{k}\right)$ converges. Then $\sum b_{k}=\sum\left(b_{k}^{\prime}-T P\left(b_{k}\right)\right)$ also has to converge. The sum $b$ of this series belongs to $D$, hence $b^{\prime}=b+T P(b)$ belongs to $(I+T)(D)$.

This example can be transferred to any infinite-dimensional Banach space $Y$ by using the results of V. M. Kadets. Let $\mathrm{SR}\left(\sum x_{i}\right)$ denote the sum range of $\sum x_{i}$ and let $X \stackrel{f}{\Rightarrow} Y$ denote the fact that the Banach space $X$ is finitely representable in the Banach space $Y$. Theorem 7.2.2 of [S91] states:

Let $X$ and $Y$ be Banach spaces, $X \stackrel{f}{\Rightarrow} Y$. Suppose that $X$ has a basis $\left\{e_{k}\right\}_{k=1}^{\infty}$ and let $\sum_{k=1}^{\infty} x_{k}$ be a series in $X$ such that $\operatorname{SR}\left(\sum_{k=1}^{\infty} x_{k}\right)$ is not a linear set. Then for any monotone sequence $\left\{a_{k}\right\}_{k=1}^{\infty}$ of positive numbers with $a_{k} \rightarrow \infty$ as $k \rightarrow \infty$, there exists a series $\sum_{k=1}^{\infty} y_{k}$ in $Y$ such that $\operatorname{SR}\left(\sum_{k=1}^{\infty} y_{k}\right)$ is not a linear set and $\left\|y_{k}\right\| \leq a_{k}\left\|x_{k}\right\|$ for all $k \in \mathbb{N}$.

Corollary 7.2.1 points out that if $X$ is $l_{2}$ then by Dvoretzky's theorem $X \stackrel{\mathrm{f}}{\Rightarrow} Y$, and Corollary 7.2.2 states that in any infinite-dimensional Banach space there are series whose sum range consists of two points. This is achieved by applying the two-point example in $L_{2}$ to Corollary 7.2.1 and following the proof of Theorem 7.2.2 to see that no new points appear and all the old ones are transferred to the space $Y$. We have an $n$-point example in $L_{2}$ which can be in the same manner, through obvious modifications in the 
proof of Theorem 7.2.2 transferred to any Banach space $Y$. Finally, for any finite-dimensional subspaces $H_{1}, H_{2}$ of an infinite-dimensional Banach space $Y$ and any isomorphism $f: H_{1} \rightarrow H_{2}$ there exists an isomorphism $\tilde{f}: Y \rightarrow Y$ extending $f$. Thus having any $n$ points satisfying some linear equations as a sum range of $y_{k}$ in $Y$ we can take an $f$ transferring them to any other $n$ points satisfying the same linear equations and then transfer the whole series by $\tilde{f}$.

Acknowledgements. I would like to thank my father, who supported me in writing this paper and was its first reader. My thanks are also due to the anonymous referee, who put a lot of work into making it better and suggested the proof of Theorem 6.2. Finally, I would like to thank Marta Szumańska, who first suggested the problem to me.

\section{References}

[S91] M. I. Kadets and V. M. Kadets, Series in Banach Spaces. Conditional and Unconditional Convergence, Birkhäuser, 1991.

[O89] M. I. Kadets and K. Woźniakowski, On series whose permutations have only two sums, Bull. Pol. Acad. Sci. Math. 37 (1989), 15-21.

[K90] V. M. Kadets, How many points can the sum-set of a series in a Banach space contain?, Teor. Funkts. Funktsional. Anal. i Prilozhen. 54 (1990), 54-57 (in Russian).

[U02] P. L. Ulyanov, On interconnections between the research of Russian and Polish mathematicians in the theory of functions, in: Banach Center Publ. 56, Inst. Math., Polish Acad. Sci., 2002, 122-128.

Department of Mathematics,

Computer Science and Mechanics

University of Warsaw

Banacha 2

02-097 Warszawa, Poland

E-mail: onufry@duch.mimuw.edu.pl

Received March 16, 2005

Revised version July 2, 2005 